\title{
TITLE:
}

\section{Ecological characteristics of recorded species}

\section{$\operatorname{AUTHOR}(\mathrm{S})$ :}

OHGAKI, SHUN-ICHI; KOMEMOTO, KEN-ICHI; FUNAYAMA, NOBUTAKA

\section{CITATION:}

OHGAKI, SHUN-ICHI ...[et al]. 4. Ecological characteristics of recorded species.

Publications of the Seto Marine Biological Laboratory. Special Publication Series 2011, 11 : 24-30

ISSUE DATE:

2011

URL:

http://hdl.handle.net/2433/159496

RIGHT: 
4. Ecological characteristics of recorded species

Table 1.

Summary of the biological characteristics of each species. SC, species code (see List of recorded species, Section 2); HT, height; WF, water flow; PL; pool. AG algae.

Significance of correlation (semi-partial correlation; Cohen et al. 2003) between the frequency of occurrence of each species (Fig. 3) and environmental variables (HT, Table 4; WF, Table 5; PL, Table 6; AG, Table 7) are shown $(++$, strong positive correlation, $\mathrm{P}<$ $0.01 ;+$, positive correlation, $\mathrm{P}<0.05 ;--$, strong negative correlation, $\mathrm{P}<0.01$; negative correlation, $\mathrm{P}<0.05)$. GR, geographic range of species. Each species was assigned to one of the four categories, i.e., $\mathrm{N}$ (northern species), $\mathrm{S}$ (southern species), $\mathrm{E}$ (extended-range species), and L (limited-range species). The ranges of these groups along the Pacific coast of Japan are: $\mathrm{N},>35^{\circ} \mathrm{N} ; \mathrm{S},<35^{\circ} \mathrm{N}$; E, from $<30^{\circ} \mathrm{N}$ to $>35^{\circ} \mathrm{N} ; \mathrm{L}, 30-35^{\circ} \mathrm{N}$ (Higo et al. 1999, Okutani 2000). MG, morphologic groups including three categories, i.e., Lp (limpet-form species), Sn (snail-form species), and Se (sedentary species). FH is feeding habit, which is shown as $\mathrm{H}$ (herbivore), $\mathrm{P}$ (predator or parasite), D (detritivore), $\mathrm{S}$ (scavenger), or F (filter feeder). An asterisk indicates that the reference information (Ref) belongs to a different species that is in the same genus as the species concerned. DV, pattern of development exhibited as Tp (totally planktonic development, i.e., larvae experience planktonic life after external fertilization), Pp (partially planktonic development, i.e., veliger larvae hatch from a mass of laid eggs), Dd (direct development, i.e., juveniles crawl out from adult females or laid eggs), or Pl (planktonic development, i.e., either Tp or Pp but exact information is unknown). References for development are shown as numerals in the right-hand column (Ref). The intensity of harvesting by local people (HV) is shown with a qualitative evaluation of ++ (heavy) or + (light), based on the observations of the authors in the study area from 1985 to 2010. References for the feeding habit and development of each species are as follows (for details, see References in Section 9). 
1) Abe (1980a), 2) Abe (1980b), 3) Amemiya (1928), 4) Amio (1963), 5) Braley (1982), 6) Bullock \& Harper (1994), 7) Fretter \& Graham (1994), 8) Habe (1943), 9) Habe (1956), 10) Hadfield et al. (1972), 11) Hawkins et al. (1998), 12) Hayes (1983), 13) Hirano (1981), 14) Hirano (2000), 15) Houbrick (1985), 16) Houbrick (1992), 17) Houbrick (1993), 18) Hughes (1974), 19) Ishida (2001), 20) Ishida (2003), 21) Iwasaki (1993), 22) Iwasaki (1996), 23) Iwasaki (2005), 24) Iwata (1952), 25) Iwata (1999), 26) Katagiri \& Katagiri (2007), 27) Kato T personal communication, 28) Kizaki (1987), 29) Kohn (1970), 30) Kohn \& Nybakken (1975), 31) Koike K personal communication, 32) Kojima (1957), 33) Kubota (2006), 34) Kuroda et al. (1957), 35) Kuwamura et al. (1983), 36) Maeda (1986), 37) Masuda (2007), 38) Matsunaga (1964), 39) McLean (1962), 40) Miyazaki (1935), 41) Miyazaki (1938), 42) Morton (1956), 43) Niina \& Kubota (2009), 44) Nishihama et al. (1986), 45) Ohgaki (1997), 46) Ohgaki (2005), 47) Ohgaki personal observation, 48) Omi \& Kuramochi (2001), 49) Ostergaard (1950), 50) Ota \& Tokeshi (2000), 51) Pastoureaud et al. (1996), 52) Robertson (1970), 53) Rosewater (1970), 54) Satomori (1982), 55) Senawong (1971), 56) Steneck \& Watling (1982), 57) Sumikawa (1963), 58) Switzer-Dunlap (1978), 59) Takada (1996), 60) Takada (2001), 61) Takada (2003), 62) Taki \& Saito (1999), 63) Tanaka (1957), 64) Tanaka (1960), 65) Tanaka (1970), 66) Taylor (1976), 67) Usuki (1970), 68) Usuki H personal communication, 69) Wada (1986), 70) Webber (1977), 71) Yamaguchi (1992), 72) Yamamoto (1997), 73) Yonge \& Thompson (1976), 74) Yoshida (1953), 75) Yoshida (1960), 76) Yoshioka (1988), 77) Yukihira et al. (1995), 78) Yusa Y. personal communication 


\begin{tabular}{|c|c|c|c|c|c|c|c|c|c|c|c|}
\hline \multicolumn{12}{|c|}{ Table $1-1$} \\
\hline $\mathrm{SC}$ & $\mathrm{HT}$ & WF & $\mathrm{PL}$ & $\mathrm{AG}$ & GR & MG & $\mathrm{FH}$ & Ref & DV & Ref & $\mathrm{HV}$ \\
\hline 1 & & & & & $\mathrm{E}$ & Lp & $\mathrm{H}$ & 6 & & & \\
\hline 2 & -- & - & & & $\mathrm{E}$ & Lp & $\mathrm{H}$ & 6 & & & \\
\hline 3 & & & & & $\mathrm{~N}$ & Lp & $\mathrm{H}, \mathrm{P}^{*}$ & 39 & & & \\
\hline 4 & - & & & & $\mathrm{E}$ & Lp & $\mathrm{H}, \mathrm{P}$ & 44 & $\mathrm{Tp}$ & 76 & + \\
\hline 5 & & & & & $\mathrm{~S}$ & Lp & $\mathrm{H}^{*}$ & 56 & & & \\
\hline 6 & & ++ & & & $\mathrm{L}$ & Lp & & & & & \\
\hline 7 & - & & & & $\mathrm{E}$ & Lp & $\mathrm{H}^{*}$ & 56 & & & \\
\hline 8 & & ++ & & & $\mathrm{L}$ & Lp & $\mathrm{H}^{*}$ & 56 & & & \\
\hline 9 & & & & & $\mathrm{E}$ & Lp & $\mathrm{H}$ & 62 & & & \\
\hline 10 & & ++ & & & $\mathrm{S}$ & $\mathrm{Lp}$ & $\mathrm{H}$ & 21 & & & \\
\hline 11 & & & & & $\mathrm{E}$ & $\mathrm{Lp}$ & $\mathrm{H}^{*}$ & 21,56 & $\mathrm{Tp}$ & 4 & \\
\hline 12 & & & & & $\mathrm{~L}$ & Lp & $\mathrm{H}_{4}$ & 13 & & & + \\
\hline 13 & - & & & & $\mathrm{E}$ & Lp & $\mathrm{H}$ & 13,21 & $\mathrm{Tp}$ & 4 & \\
\hline 14 & & - & + & & $\mathrm{E}$ & Lp & $\mathrm{H}$ & 13,60 & $\mathrm{Tp}$ & 4 & \\
\hline 15 & & -- & & & $\mathrm{S}$ & Lp & $\mathrm{H}_{4}$ & 13 & $\mathrm{Tp}$ & 4 & \\
\hline 16 & & ++ & & & $\mathrm{L}$ & Lp & $\mathrm{H}^{*}$ & 56 & & 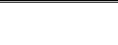 & \\
\hline 17 & & & & & $\mathrm{E}$ & Lp & $\mathrm{H}^{*}$ & 56 & $\mathrm{Tp}$ & 4 & \\
\hline 18 & & ++ & & & $\mathrm{E}$ & Lp & $\mathrm{H}^{*}$ & 56 & & & \\
\hline 19 & & 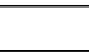 & & & $\mathrm{N}$ & Lp & $\mathrm{H}^{*}$ & 56 & & & \\
\hline 20 & & - & & + & $\mathrm{E}$ & Lp & $\mathrm{H}^{*}$ & 60 & $\mathrm{Tp}$ & 4 & \\
\hline 21 & -- & -- & & & $\mathrm{L}$ & $\mathrm{Lp}$ & $\mathrm{H}^{*}$ & 60 & & & \\
\hline 22 & & & ++ & & $\mathrm{N}$ & $\mathrm{Lp}$ & $\mathrm{H}$ & 60 & & & \\
\hline 23 & -- & -- & ++ & & $\mathrm{N}$ & $\mathrm{Lp}$ & $\mathrm{H}$ & 60 & $\mathrm{Tp}$ & 4 & \\
\hline 24 & & & & & $\mathrm{~N}$ & $\mathrm{Lp}$ & $\mathrm{H}$ & 34 & $\mathrm{Tp}$ & 4 & + \\
\hline 25 & - & & & & $\mathrm{E}$ & $\mathrm{Lp}$ & $\mathrm{H}$ & 23 & $\mathrm{Tp}$ & 4 & ++ \\
\hline 26 & & ++ & & & Sk & Lp & $\mathrm{H}^{*}$ & 23,56 & & & ++ \\
\hline 27 & & ++ & & & $\mathrm{E}$ & $\mathrm{Lp}$ & & & & & \\
\hline 28 & - & & & & $\mathrm{L}$ & Lp & & & & & \\
\hline 29 & -- & & & & $\mathrm{N}$ & Lp & & & & & \\
\hline 30 & & & & & $\mathrm{~N}$ & Lp & & & & & \\
\hline 31 & & & & & $\mathrm{~N}$ & Sn & $\mathrm{H}$ & 23 & & & + \\
\hline 32 & & & & & $\mathrm{~N}$ & $\mathrm{Sn}$ & $\mathrm{H}$ & 23 & & & + \\
\hline 33 & & & & & $\mathrm{E}$ & $\mathrm{Sn}$ & $\mathrm{H}^{*}$ & 23 & & & + \\
\hline 34 & & & & + & $\mathrm{S}$ & $\mathrm{Sn}$ & & & & & + \\
\hline 35 & & & & & $\mathrm{~N}$ & $\mathrm{Sn}$ & & & & & + \\
\hline 36 & & & + & & $\mathrm{N}$ & $\mathrm{Sn}$ & $\mathrm{H}$ & 23 & & & + \\
\hline 37 & & & & & $\mathrm{Sk}$ & $\mathrm{Sn}$ & & & & & + \\
\hline 38 & & ++ & & ++ & Sk & $\mathrm{Sn}$ & & & & & + \\
\hline 39 & & + & & & $\mathrm{S}$ & Sn & & & & & + \\
\hline 40 & & & & & $\mathrm{Sk}$ & $\mathrm{Sn}$ & & & & & + \\
\hline 41 & -- & & & & $\mathrm{S}$ & $\mathrm{Sn}$ & & & & & ++ \\
\hline 42 & & & & & $\mathrm{~S}$ & $\mathrm{Sn}$ & & & & & \\
\hline 43 & & & - & & $\mathrm{N}$ & $\mathrm{Sn}$ & & & & & \\
\hline 44 & ++ & & & & $\mathrm{N}$ & $\mathrm{Sn}$ & $\mathrm{H}$ & 23,60 & $\mathrm{Tp}$ & 57,59 & \\
\hline 45 & & & & & $\mathrm{~N}$ & $\mathrm{Sn}$ & $\mathrm{H}^{*}$ & 23,56 & & & \\
\hline 46 & & & & & $\mathrm{~N}$ & $\mathrm{Sn}$ & & & & & \\
\hline 47 & & & & & $\mathrm{~N}$ & $\mathrm{Sn}$ & & & & & \\
\hline 48 & & + & & & $\mathrm{S}$ & $\mathrm{Sn}$ & & & & & \\
\hline 49 & & ++ & & + & Sk & $\mathrm{Sn}$ & & & & & \\
\hline 50 & & & & & $\mathrm{~S}$ & $\mathrm{Sn}$ & & & & & \\
\hline
\end{tabular}




\begin{tabular}{|c|c|c|c|c|c|c|c|c|c|c|c|}
\hline \multicolumn{12}{|c|}{ Table 1-2 } \\
\hline $\mathrm{SC}$ & HT & WF & PL & $\mathrm{AG}$ & GR & MG & $\mathrm{FH}$ & Ref & DV & Ref & $\mathrm{HV}$ \\
\hline 51 & & & & & Sk & $\mathrm{Sn}$ & & & & & \\
\hline 52 & & ++ & & & $\mathrm{N}$ & $\mathrm{Sn}$ & $\mathrm{P}$ & 23,27 & & & \\
\hline 53 & & & & & $\mathrm{~N}$ & $\mathrm{Sn}$ & & & & & \\
\hline 54 & -- & -- & & & $\mathrm{S}$ & $\mathrm{Sn}$ & & & & & \\
\hline 55 & & & & & $\mathrm{~L}$ & $\mathrm{Sn}$ & & & & & \\
\hline 56 & & & & & $\mathrm{~L}$ & Sn & & & & & \\
\hline 57 & -- & -- & & & $\mathrm{S}$ & $\mathrm{Sn}$ & & & & & \\
\hline 58 & -- & -- & & & $\mathrm{L}$ & $\mathrm{Sn}$ & & & & & \\
\hline 59 & & 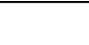 & & & $\mathrm{S}$ & $\mathrm{Sn}$ & & & & & \\
\hline 60 & & -- & + & & $\mathrm{N}$ & $\mathrm{Sn}$ & $\mathrm{H}$ & 23,77 & $\mathrm{Tp}$ & 4 & + \\
\hline 61 & -- & & & & $\mathrm{S}$ & $\mathrm{Sn}$ & $\mathrm{H}^{*}$ & 23,56 & & & \\
\hline 62 & - & & & & $\mathrm{S}$ & $\mathrm{Sn}$ & & & & & \\
\hline 63 & & - & & & $\mathrm{S}$ & $\mathrm{Sn}$ & $\mathrm{H}$ & 23 & $\mathrm{Tp}$ & 23,31 & \\
\hline 64 & ++ & & & & $\mathrm{L}$ & $\mathrm{Sn}$ & $\mathrm{Hi}$ & 60 & $\overline{\mathrm{Dd}}$ & 2 & \\
\hline 65 & & & & & $\mathrm{Sk}$ & $\mathrm{Sn}$ & $\mathrm{H}^{*}$ & 23,56 & $\mathrm{Tp}$ & 31 & \\
\hline 66 & & & & & $\mathrm{~L}$ & $\mathrm{Sn}$ & & & & & \\
\hline 67 & & & & & $\mathrm{Sk}$ & $\mathrm{Sn}$ & $\mathrm{H}, \mathrm{D}^{*}$ & 16 & & & \\
\hline 68 & & & & & $\mathrm{Sk}$ & Sn & & & & & \\
\hline 69 & & & & & $\mathrm{~L}$ & $\mathrm{Sn}$ & & & & & \\
\hline 70 & & & ++ & & $\mathrm{S}$ & $\mathrm{Sn}$ & $\mathrm{H}, \mathrm{D}$ & 15,36 & $\mathrm{Pp}$ & 15 & \\
\hline 71 & & & ++ & & $\mathrm{E}$ & $\mathrm{Sn}$ & & & $\mathrm{Dd}$ & 17 & \\
\hline 72 & & & ++ & & $\mathrm{S}$ & $\mathrm{Sn}$ & & & & & \\
\hline 73 & + & & & & $\mathrm{S}$ & $\mathrm{Sn}$ & & & $\mathrm{Pp}$ & 45 & \\
\hline 74 & & & & & $\mathrm{~S}$ & $\mathrm{Sn}$ & & & & & \\
\hline 75 & & & & & $\mathrm{Sk}$ & $\mathrm{Sn}$ & & & $\mathrm{Tp}$ & 4,49 & \\
\hline 76 & & & & & $\mathrm{~S}$ & $\mathrm{Sn}$ & & & $\mathrm{Tp}$ & 53 & \\
\hline 77 & ++ & & & & $\mathrm{E}$ & $\mathrm{Sn}$ & & & $\mathrm{Tp}$ & 4,9 & \\
\hline 78 & ++ & & & & $\mathrm{N}$ & $\mathrm{Sn}$ & $\mathrm{H}$ & 46 & $\mathrm{Tp}$ & 4,9 & \\
\hline 79 & ++ & & & & $\mathrm{S}$ & $\mathrm{Sn}$ & $\mathrm{H}^{*}$ & 46 & $\mathrm{Tp}$ & 4,9 & \\
\hline 80 & ++ & & & & $\mathrm{S}$ & $\mathrm{Sn}$ & $\mathrm{H}^{*}$ & 46 & $\mathrm{Tp}$ & 4,9 & \\
\hline 81 & ++ & & & & $\mathrm{E}$ & $\mathrm{Sn}$ & $\mathrm{Hu}$ & 28,61 & $\mathrm{Tp}$ & 4,32 & \\
\hline 82 & & & ++ & & $\mathrm{N}$ & $\mathrm{Sn}$ & $\mathrm{H}, \mathrm{D}^{*}$ & 7,56 & & & \\
\hline 83 & & & & & $\mathrm{~L}$ & $\mathrm{Sn}$ & & & & & \\
\hline 84 & & & & & $\mathrm{~S}$ & $\mathrm{Sn}$ & $\mathrm{H}, \mathrm{D}$ & 69 & $\mathrm{Pp}$ & 35 & ++ \\
\hline 85 & & & & & $\mathrm{~S}$ & $\mathrm{Sn}$ & $\mathrm{H}$ & 36 & & & \\
\hline 86 & -- & & & & $\mathrm{E}$ & $\mathrm{Se}$ & $\mathrm{H}, \mathrm{P}, \mathrm{D}$ & 23,38 & $\mathrm{Dd}$ & 4 & \\
\hline 87 & -- & & & & $\mathrm{S}$ & $\mathrm{Se}$ & & & & & \\
\hline 88 & $3--$ & (-- & & & $\mathrm{S}$ & $\mathrm{Se}$ & & & & & \\
\hline 89 & & & & + & $\mathrm{S}$ & $\mathrm{Se}$ & $\mathrm{F}^{*}$ & 7,73 & $\mathrm{Dd}$ & 4 & \\
\hline 90 & & & & & $\mathrm{~N}$ & $\mathrm{Se}$ & $\mathrm{F}$ & 73 & $\mathrm{Pl}$ & 73 & \\
\hline 91 & & ++ & & & $\mathrm{L}$ & $\mathrm{Se}$ & $\mathrm{F}^{*}$ & 10,18 & & & \\
\hline 92 & & & & & Sk & $\mathrm{Se}$ & $\mathrm{F}^{*}$ & 10,18 & & & \\
\hline 93 & & & & & $\mathrm{~L}$ & $\mathrm{Se}$ & $\mathrm{F}^{*}$ & 20,36 & & & \\
\hline 94 & - & & & & $\mathrm{N}$ & $\mathrm{Se}$ & $\mathrm{Fi}$ & 20,36 & $\mathrm{Pp}$ & 4 & \\
\hline 95 & & & & & $\mathrm{~S}$ & $\mathrm{Sn}$ & $\mathrm{H}$ & 36,71 & & & \\
\hline 96 & & & & & $\mathrm{~S}$ & $\mathrm{Sn}$ & & & & & \\
\hline 97 & & & & & $\mathrm{~S}$ & $\mathrm{Sn}$ & & & & & \\
\hline 98 & & & & & $\mathrm{~S}$ & $\mathrm{Sn}$ & & & & & \\
\hline 99 & & + & & & $\mathrm{S}$ & $\mathrm{Sn}$ & $\mathrm{H}>\mathrm{P}$ & 48,71 & $\mathrm{Pp}$ & 49 & \\
\hline 100 & & & & & $\mathrm{~S}$ & $\mathrm{Sn}$ & & & & & \\
\hline
\end{tabular}




\begin{tabular}{|c|c|c|c|c|c|c|c|c|c|c|c|}
\hline \multicolumn{12}{|c|}{ Table 1-3 } \\
\hline $\mathrm{SC}$ & $\mathrm{HT}$ & WF & PL & $\mathrm{AG}$ & GR & MG & $\mathrm{FH}$ & Ref & DV & Ref & $\mathrm{HV}$ \\
\hline 101 & & & & & $\mathrm{~S}$ & $\mathrm{Sn}$ & & & & & \\
\hline 102 & & & & & $\mathrm{~S}$ & $\mathrm{Sn}$ & & & & & \\
\hline 103 & & & & & $\mathrm{E}$ & $\mathrm{Sn}$ & $\mathrm{P}>\mathrm{DH}$ & 23 & & & \\
\hline 104 & & & & & $\mathrm{~S}$ & $\mathrm{Sn}$ & $\mathrm{P}>\mathrm{H}$ & 12 & $\mathrm{Pp}$ & 49 & \\
\hline 105 & & & & & $\mathrm{~S}$ & $\mathrm{Sn}$ & & & & & \\
\hline 106 & & & & & $\mathrm{~S}$ & $\mathrm{Sn}$ & & & & & \\
\hline 107 & & ++ & & & $\mathrm{S}$ & $\mathrm{Sn}$ & & & & & \\
\hline 108 & & & & & $\mathrm{~S}$ & $\mathrm{Sn}$ & $\mathrm{H}$ & $71 \mid$ & & & \\
\hline 109 & & & & & $\mathrm{~S}$ & $\mathrm{Sn}$ & & & $\mathrm{Pp}$ & 49 & \\
\hline 110 & & & & & $\mathrm{~S}$ & $\mathrm{Sn}$ & & & & & \\
\hline 111 & & & & & $\mathrm{~S}$ & $\mathrm{Sn}$ & $\mathrm{P}^{*}$ & 7 & & & \\
\hline 112 & & & & & $\mathrm{~L}$ & $\mathrm{Sn}$ & $\mathrm{P}^{* 1}$ & 7 & & & \\
\hline 113 & & & & & $\mathrm{~S}$ & $\mathrm{Sn}$ & $\mathrm{P*}$ & 73 & & & \\
\hline 114 & & & & & $\mathrm{~S}$ & $\mathrm{Sn}$ & $\mathrm{PI}$ & 36 & & & \\
\hline 115 & $1--$ & - & & & $\mathrm{S}$ & $\mathrm{Sn}$ & & & & & \\
\hline 116 & -- & - & & & $\mathrm{L}$ & $\mathrm{Sn}$ & $\mathrm{P}^{*}$ & 7,52 & & & \\
\hline 117 & & & & & $\mathrm{~N}$ & $\mathrm{Sn}$ & $\mathrm{P}^{*}$ & 7,52 & & & \\
\hline 118 & & & & & $\mathrm{~L}$ & $\mathrm{Sn}$ & $\mathrm{P}$ & 8 & & & \\
\hline 119 & -- & 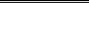 & & & $\mathrm{N}$ & $\mathrm{Sn}$ & $\mathrm{P}^{*}$ & 7 & & & \\
\hline 120 & & 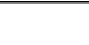 & & & $\mathrm{L}$ & $\mathrm{Sn}$ & $\mathrm{P}^{*}$ & 7 & & & \\
\hline 121 & & 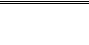 & & & $\mathrm{S}$ & $\mathrm{Sn}$ & & & & & \\
\hline 122 & -- & -- & & & $\mathrm{S}$ & $\mathrm{Sn}$ & $\mathrm{P}>\mathrm{S}$ & 1,19 & & & \\
\hline 123 & -- & -- & & & $\mathrm{E}$ & $\mathrm{Sn}$ & $\mathrm{P}, \mathrm{S}$ & 1,19 & & & \\
\hline 124 & 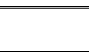 & - & & & $\mathrm{Sk}$ & $\mathrm{Sn}$ & $\mathrm{P}$ & 1 & Dd & 72 & \\
\hline 125 & - & - & & & $\mathrm{S}$ & $\mathrm{Sn}$ & $\mathrm{P}^{*}$ & 1 & & & \\
\hline 126 & & & & & $\mathrm{Sk}$ & $\mathrm{Sn}$ & & & & & \\
\hline 127 & & & & & $\mathrm{Sk}$ & $\mathrm{Sn}$ & $\mathrm{P}^{*}$ & 52,66 & & & \\
\hline 128 & & & & & Sk & $\mathrm{Sn}$ & $\mathrm{PI}$ & 66 & & & \\
\hline 129 & -- & & & & $\mathrm{S}$ & $\mathrm{Sn}$ & $\mathrm{P}^{*}$ & 1,66 & & & \\
\hline 130 & - & & & & $\mathrm{S}$ & $\mathrm{Sn}$ & $\mathrm{P}$ & 1 & & & \\
\hline 131 & -- & -- & & & $\mathrm{Sk}$ & $\mathrm{Sn}$ & $\mathrm{P}^{*}$ & 1,66 & & & \\
\hline 132 & -- & - & & & $\mathrm{S}$ & $\mathrm{Sn}$ & $\mathrm{P}$ & 1,19 & & & \\
\hline 133 & & & & & Sk & $\mathrm{Sn}$ & $\mathrm{P*}$ & 1,66 & & & \\
\hline 134 & & & & & $\mathrm{~S}$ & $\mathrm{Sn}$ & & & & & \\
\hline 135 & & & & & $\mathrm{~S}$ & $\mathrm{Sn}$ & $\mathrm{P}$ & 66 & & & \\
\hline 136 & & & & & $\mathrm{Sk}$ & $\mathrm{Sn}$ & $\mathrm{P*}$ & 66 & & & + \\
\hline 137 & & & & & $\mathrm{~S}$ & $\mathrm{Sn}$ & $\mathrm{P}^{*}$ & 66 & & & \\
\hline 138 & & ++ & & - & $\mathrm{Sk}$ & $\mathrm{Sn}$ & $\mathrm{P}^{*}$ & 66 & & & + \\
\hline 139 & & & & & $\mathrm{Sk}$ & $\mathrm{Sn}$ & $\mathrm{P}$ & 66 & & & + \\
\hline 140 & & & & & Sk & $\mathrm{Sn}$ & $\mathrm{P}$ & 66 & & & \\
\hline 141 & $1--$ & & & & $\mathrm{N}$ & $\mathrm{Sn}$ & $\mathrm{P}$ & 1,23 & $\mathrm{Pp}$ & 4 & + \\
\hline 142 & -- & & & & $\mathrm{N}$ & $\mathrm{Sn}$ & $\mathrm{P}$ & 1,23 & $\mathrm{Pp}$ & 4 & + \\
\hline 143 & $1--$ & & & & $\mathrm{E}$ & $\mathrm{Sn}$ & $\mathrm{Pi}$ & 1 & & & + \\
\hline 144 & 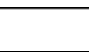 & & & & $\mathrm{S}$ & $\mathrm{Sn}$ & $\mathrm{P}^{*}$ & 52,66 & & & \\
\hline 145 & -- & & & & $\mathrm{S}$ & $\mathrm{Sn}$ & $\mathrm{H}$ & 68 & Dd & 4 & \\
\hline 146 & - & & & & $\mathrm{L}$ & $\mathrm{Sn}$ & $\mathrm{H}>\mathrm{S}, \mathrm{D}$ & 23 & Dd & 4 & \\
\hline 147 & & & & & $\mathrm{~S}$ & $\mathrm{Sn}$ & & & & & \\
\hline 148 & & & & & L & $\mathrm{Sn}$ & & & & & \\
\hline 149 & & & & & $\mathrm{E}$ & $\mathrm{Sn}$ & & & $\mathrm{Pp}$ & 4 & \\
\hline 150 & & & & & $\mathrm{~N}$ & $\mathrm{Sn}$ & & & & & \\
\hline
\end{tabular}




\begin{tabular}{|c|c|c|c|c|c|c|c|c|c|c|c|}
\hline \multicolumn{12}{|c|}{ Table 1-4 } \\
\hline $\mathrm{SC}$ & HT & WF & $\mathrm{PL}$ & $\mathrm{AG}$ & GR & MG & $\mathrm{FH}$ & Ref & DV & Ref & $\mathrm{HV}$ \\
\hline 151 & & & & & $\mathrm{~S}$ & $\mathrm{Sn}$ & & & & & \\
\hline 152 & & & & & $\mathrm{E}$ & $\mathrm{Sn}$ & & & $\mathrm{Pp}$ & 4 & \\
\hline 153 & & & & & $\mathrm{Sk}$ & $\mathrm{Sn}$ & & & & & \\
\hline 154 & & & & & $\mathrm{E}$ & $\mathrm{Sn}$ & & & & & \\
\hline 155 & & & ++ & & $\mathrm{S}$ & $\mathrm{Sn}$ & & & & & \\
\hline 156 & & & ++ & & $\mathrm{N}$ & $\mathrm{Sn}$ & & & & & \\
\hline 157 & & & & & $\mathrm{~L}$ & $\mathrm{Sn}$ & & & & & \\
\hline 158 & & & & & $\mathrm{~S}$ & $\mathrm{Sn}$ & $\mathrm{P}, \mathrm{S}$ & 23,50 & & & \\
\hline 159 & & & & & $\mathrm{~S}$ & $\mathrm{Sn}$ & $\mathrm{P}$ & 29 & & & \\
\hline 160 & & & & & $\mathrm{Sk}$ & $\mathrm{Sn}$ & $\mathrm{P}^{*}$ & 29 & & & \\
\hline 161 & -- & -- & & & $\mathrm{S}$ & $\mathrm{Sn}$ & $\mathrm{P}^{*}$ & 29 & & & \\
\hline 162 & & & & + & $\mathrm{Sk}$ & $\mathrm{Sn}$ & $\mathrm{P}$ & 30 & & & \\
\hline \begin{tabular}{|l|}
163 \\
\end{tabular} & & & & & $\mathrm{E}$ & $\mathrm{Sn}$ & $\mathrm{P}$ & 30 & & & \\
\hline 164 & & & & & $\mathrm{Sk}$ & $\mathrm{Sn}$ & $\mathrm{PI}$ & 30 & $\mathrm{Pp}$ & 70 & \\
\hline 165 & & & & & $\mathrm{~S}$ & $\mathrm{Sn}$ & $\mathrm{P}$ & 30 & & & \\
\hline 166 & & & & & Sk & $\mathrm{Sn}$ & $\mathrm{P}$ & 30 & & & \\
\hline 167 & & & & & $\mathrm{Sk}$ & $\mathrm{Sn}$ & $\mathrm{P}$ & 30 & & & \\
\hline 168 & & & & & $\mathrm{~L}$ & $\mathrm{Sn}$ & i & & & & \\
\hline 169 & & & & & Sk & $\mathrm{Sn}$ & 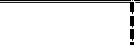 & & & & \\
\hline 170 & & & & & $\mathrm{~L}$ & $\mathrm{Sn}$ & $\mathrm{P}$ & 25 & & & \\
\hline 171 & & & & & $\mathrm{~N}$ & $\mathrm{Sn}$ & $\mathrm{P}$ & 25 & & & \\
\hline 172 & & & & & $\mathrm{~S}$ & $\mathrm{Sn}$ & & & & & \\
\hline 173 & -- & -- & & & $\mathrm{E}$ & $\mathrm{Sn}$ & $\mathrm{P}$ & 33 & $\mathrm{Pp}$ & 49 & \\
\hline 174 & & & & & $\mathrm{~S}$ & $\mathrm{Sn}$ & & & & & \\
\hline 175 & & & & & $\mathrm{~N}$ & $\mathrm{Sn}$ & $\mathrm{H}$ & 14 & $\mathrm{Pp}$ & 4 & \\
\hline 176 & & & & & $\mathrm{~S}$ & $\mathrm{Sn}$ & & & & & \\
\hline 177 & & & & & $\mathrm{~S}$ & $\mathrm{Sn}$ & & & & & \\
\hline 178 & & & & & $\mathrm{~N}$ & $\mathrm{Sn}$ & & & $\mathrm{Pp}$ & 4 & \\
\hline 179 & & & & & $\mathrm{~S}$ & $\mathrm{Sn}$ & $\mathrm{H}^{*}$ & 14 & $\mathrm{Pp}$ & 58 & \\
\hline 180 & -- & - & & & $\mathrm{E}$ & $\mathrm{Sn}$ & $\mathrm{H}$ & \begin{tabular}{|l|}
14,67 \\
\end{tabular} & $\mathrm{Pp}$ & 58,78 & \\
\hline 181 & -- & -- & & & $\mathrm{E}$ & $\mathrm{Sn}$ & $\mathrm{HI}$ & 14,68 & $\mathrm{Pp}$ & 4,78 & \\
\hline 182 & -- & -- & & & $\mathrm{S}$ & $\mathrm{Sn}$ & $\mathrm{H}, \mathrm{S}$ & 23 & $\mathrm{Pp}$ & 78 & \\
\hline 183 & & & & & $\mathrm{E}$ & $\mathrm{Sn}$ & $\mathrm{H}$ & 14,23 & $\mathrm{Pp}$ & 4,58 & \\
\hline 184 & & & & & $\mathrm{~S}$ & $\mathrm{Sn}$ & & & $\mathrm{Pp}$ & 4,58 & \\
\hline 185 & & & & & $\mathrm{~L}$ & $\mathrm{Sn}$ & $\mathrm{P}$ & 14 & & & \\
\hline 186 & & & & & $\mathrm{~S}$ & $\mathrm{Sn}$ & & & & & \\
\hline 187 & & & & & $\mathrm{~L}$ & $\mathrm{Sn}$ & $\mathrm{P}>\mathrm{H}, \mathrm{D}$ & 14,23 & $\mathrm{Pp}$ & 43 & \\
\hline 188 & & & & & $\mathrm{~S}$ & $\mathrm{Sn}$ & $\mathrm{P}$ & 14 & & & \\
\hline 189 & & & & & $\mathrm{~S}$ & $\mathrm{Sn}$ & $\mathrm{P}$ & 14 & & & \\
\hline 190 & & & & & $\mathrm{~S}$ & $\mathrm{Sn}$ & $\mathrm{Pi}$ & 14 & & & \\
\hline \begin{tabular}{|l|}
191 \\
\end{tabular} & & & & & $\mathrm{~S}$ & $\mathrm{Sn}$ & & & & & \\
\hline 192 & & & & & $\mathrm{E}$ & $\mathrm{Sn}$ & $\mathrm{P}>\mathrm{D}$ & 14,23 & & & \\
\hline \begin{tabular}{|l|}
193 \\
\end{tabular} & & & & & $\mathrm{~S}$ & $\mathrm{Sn}$ & $\mathrm{Pi}$ & 37 & Dd & 43 & \\
\hline 194 & & & & & $\mathrm{~S}$ & $\mathrm{Sn}$ & $\mathrm{P}$ & 14 & $\mathrm{Pp}$ & 49 & \\
\hline 195 & & & & & $?$ & $\mathrm{Sn}$ & & & & & \\
\hline 196 & & & & & $\mathrm{~L}$ & $\mathrm{Sn}$ & & & & & \\
\hline 197 & & & & & $\mathrm{~L}$ & $\mathrm{Sn}$ & & & Dd & 26 & \\
\hline 198 & ++ & & & & $\mathrm{L}$ & $\mathrm{Sn}$ & $\mathrm{H}^{*}$ & 21 & & & \\
\hline 199 & & & - & & $\mathrm{N}$ & $\mathrm{Sn}$ & $\mathrm{Hi}$ & 13 & $\mathrm{Pp}$ & 4 & \\
\hline 200 & - & & & & $\mathrm{N}$ & $\mathrm{Sn}$ & $\mathrm{H}$ & 21 & $\mathrm{Pp}$ & 4 & \\
\hline
\end{tabular}




\begin{tabular}{|c|c|c|c|c|c|c|c|c|c|c|c|}
\hline \multicolumn{12}{|c|}{ Table $1-5$} \\
\hline $\mathrm{SC}$ & $\begin{array}{l}\mathrm{HT} \\
\end{array}$ & $\overline{\mathrm{WF}}$ & $\begin{array}{l}\mathrm{PL} \\
\end{array}$ & $\overline{\mathrm{AG}}$ & GR & MG & $\mathrm{FH}$ & Ref & DV & Ref & $\mathrm{HV}$ \\
\hline 201 & & & & & $\mathrm{~L}$ & & $\mathrm{~F}^{*}$ & 73 & & & \\
\hline 202 & & & & & $\mathrm{E}$ & $\mathrm{Se}$ & & & & & \\
\hline 203 & & & & & $\mathrm{E}$ & $\mathrm{Se}$ & & & & & \\
\hline 204 & & & & & Sk & $\mathrm{Se}$ & & & & & \\
\hline 205 & & & & & $\mathrm{E}$ & $\mathrm{Se}$ & & & & & \\
\hline 206 & & & & & $\mathrm{~S}$ & $\mathrm{Se}$ & & & & & \\
\hline 207 & -- & & & & $\mathrm{E}$ & $\mathrm{Se}$ & & & & & ++ \\
\hline 208 & & & & & $\mathrm{~S}$ & $\mathrm{Se}$ & & & & & \\
\hline 209 & & ++ & & & $\mathrm{N}$ & $\mathrm{Se}$ & & & $\mathrm{Tp}$ & 40,74 & \\
\hline 210 & & ++ & & & $\mathrm{N}$ & $\mathrm{Se}$ & & & $\mathrm{Tp}$ & 24,57 & \\
\hline 211 & ++ & & & & $\mathrm{L}$ & $\mathrm{Se}$ & & & & & \\
\hline 212 & -- & & & & $\mathrm{S}$ & $\mathrm{Se}$ & $\mathrm{F}$ & 55 & $\mathrm{Tp}$ & 47 & \\
\hline 213 & - & & & & $\mathrm{S}$ & $\mathrm{Se}$ & & & & & \\
\hline 214 & & ++ & & & $\mathrm{N}$ & $\mathrm{Se}$ & & & $\mathrm{Tp}$ & 47 & \\
\hline 215 & & & & & $\mathrm{Sk}$ & $\mathrm{Se}$ & & & & & \\
\hline 216 & & + & & & $\mathrm{N}$ & $\mathrm{Se}$ & $\mathrm{F}$ & 55 & & & \\
\hline 217 & & & & & $\mathrm{~L}$ & $\mathrm{Se}$ & & & & & \\
\hline 218 & & + & & & Sk & $\mathrm{Se}$ & & & & & \\
\hline 219 & & & & & $\mathrm{Sk}$ & $\mathrm{Se}$ & & & $\overline{\mathrm{Pl}}$ & 65 & \\
\hline 220 & -- & & + & & $\mathrm{S}$ & $\mathrm{Se}$ & & & $\overline{\mathrm{Pl}}$ & 34,63 & \\
\hline 221 & + & & & & Sk & $\mathrm{Se}$ & & & & & \\
\hline 222 & & ++ & & & $\mathrm{S}$ & $\mathrm{Se}$ & & & & & \\
\hline 223 & & & & & $\mathrm{~S}$ & $\mathrm{Se}$ & & & & & \\
\hline 224 & & & & & $\mathrm{~S}$ & $\mathrm{Se}$ & & & $\mathrm{Tp}$ & 54 & \\
\hline 225 & & & & & $\mathrm{~S}$ & $\mathrm{Se}$ & & & & & \\
\hline 226 & & ++ & & + & $\mathrm{S}$ & $\mathrm{Se}$ & & & & & \\
\hline 227 & & & & & $\mathrm{E}$ & $\mathrm{Se}$ & $\mathrm{F}$ & 51 & $\mathrm{Tp}$ & 3 & \\
\hline 228 & & & & & $\mathrm{~N}$ & $\mathrm{Se}$ & & & $\mathrm{Tp}$ & 3 & + \\
\hline 229 & & -- & & & $\mathrm{E}$ & $\mathrm{Se}$ & & & $\mathrm{Tp}$ & 3,64 & \\
\hline 230 & & & -- & & Sk & $\mathrm{Se}$ & $\mathrm{F}$ & 11 & $\mathrm{Pl}$ & 5 & \\
\hline 231 & & & & & $\mathrm{~S}$ & $\mathrm{Se}$ & & & & & \\
\hline 232 & & & - & & $\mathrm{E}$ & & $\mathrm{F}^{*}$ & 42 & $\overline{\mathrm{Dd}}$ & 22 & \\
\hline 233 & -- & & & & $\mathrm{E}$ & $\mathrm{Se}$ & & & & & \\
\hline 234 & & & & & $\mathrm{E}$ & $\mathrm{Se}$ & & & & & \\
\hline 235 & & & & & $\mathrm{~S}$ & $\mathrm{Se}$ & & & & & \\
\hline 236 & & & & & $\mathrm{~S}$ & & & & & & \\
\hline 237 & & & & & Sk & & & & & & \\
\hline 238 & & & & & $\mathrm{~N}$ & & & & & & \\
\hline 239 & & & & & $\mathrm{~S}$ & & & & $\mathrm{Pl}$ & 75 & \\
\hline 240 & & & & & $\mathrm{E}$ & & & & & & \\
\hline 241 & & & & & $\mathrm{Sk}$ & & & & & & \\
\hline 242 & & ++ & & & $\mathrm{S}$ & $\mathrm{Se}$ & & & $\mathrm{Pl}$ & 41 & \\
\hline
\end{tabular}

